\title{
Towards an understanding of isospin violation in pion-nucleon scattering
}

\author{
Nadia Fettes* and Ulf-G. Meißner ${ }^{\dagger}$ \\ Forschungszentrum Jülich, Institut für Kernphysik (Theorie), D-52425 Jülich, Germany
}

(Received 18 August 2000; published 15 March 2001)

\begin{abstract}
We investigate isospin breaking in low-energy pion-nucleon scattering in the framework of chiral perturbation theory. This work extends the systematic analysis of Fettes et al. [Phys. Lett. B 451, 233 (1999)] to the energy range above threshold. Various relations, which identically vanish in the limit of isospin symmetry, are used to quantify isospin breaking effects. We study the energy dependence of the $S$ - and $P$-wave projections of these ratios and find dramatic effects in the $S$ waves of those two relations which are given in terms of isoscalar quantities only. This effect drops rather quickly with growing center-of-mass energy.
\end{abstract}

DOI: 10.1103/PhysRevC.63.045201

PACS number(s): 13.75.Gx, 12.39.Fe, 13.40.Ks, 24.85.+p

\section{INTRODUCTION}

Pion-nucleon scattering $(\pi N)$ is one of the prime reactions to test our understanding not only of the spontaneous and explicit chiral symmetry breaking QCD is supposed to undergo, but also of isospin symmetry violation. The pionnucleon system is particularly well suited for such an analysis, since chiral symmetry breaking and isospin breaking appear at the same chiral order. For neutral-pion scattering off nucleons, the isospin violating effects can be dramatically enhanced due to the smallness of the isoscalar pion-nucleon amplitude [2,3]. This spectacular effect in the difference of the $\pi^{0} p$ and $\pi^{0} n$ scattering lengths is, however, at present not amenable to a direct experimental verification. It is therefore mandatory to include also the channels with charged pions in any analysis of isospin violation. The immense experimental effort in the domain of low-energy pion-nucleon physics has stimulated considerable interest in using $\pi N$ scattering data to extract information about the violation of isospin symmetry of the strong interactions $[4,5]$. Some analyses indicate effects as large as 7\% [6,7]. In both these analyses, the source of this rather large effect remains mysterious. Since the two methods are independent and based on different approaches, it became a challenge to find a theoretical explanation for these rather phenomenological observations. Microscopically, there are two competing sources of isospin violation, which are generally of the same size: namely, the strong effect due to the light quark mass difference $m_{d}-m_{u} \simeq m_{u}$ and the electromagnetic one caused by virtual photons. There is thus a need to describe both effects, the strong and the electromagnetic interaction, consistently. One of the major criticisms raised against the analyses of isospin violation performed up to now is their inherent incompatibility of electromagnetic and strong effects. Chiral perturbation theory $(\chi \mathrm{PT})$ is able to remedy this problem. Ongoing effort in this field has resulted in the development of an effective field theory of pions, nucleons, and virtual photons, which now allows for the consistent separation of both sources of isospin violation. The corresponding effective Lagrangian was developed in $[8,3,9]$, extending the stan-

\footnotetext{
*Electronic address: n.fettes@fz-juelich.de

†Electronic address: u.meissner@fz-juelich.de
}

dard $\pi N$ effective field theory (EFT). The pertinent power counting of the EFT is based on the (phenomenological) observation that besides the pion mass and momenta, the electric charge $e$ should be counted as an additional small parameter, given the fact that

$$
\frac{e^{2}}{4 \pi} \simeq \frac{M_{\pi}^{2}}{\left(4 \pi F_{\pi}\right)^{2}} \simeq \frac{1}{100} .
$$

Note, however, that $M_{\pi}$ vanishes in the chiral limit, whereas the left-hand side of Eq. (1) remains finite. If this counting scheme is applied, we only have to deal with one expansion parameter $q$, which corresponds to small momenta and pion masses, as well as elementary charges. Since every emitted virtual photon will have to be reabsorbed, we will only have to consider terms proportional to the square of the charge matrix. The difference of the squares of the charged- and neutral-pion masses is thus an effect of second chiral order, i.e., a leading order effect. On the other hand, the mass difference for the nucleons also starts out at second order and is thus suppressed by two chiral orders.

The analysis of isospin violation in $\pi N$ scattering proceeds essentially in three steps. First, one ignores all isospin breaking effects; i.e., one sets $e=0$ and $m_{u}=m_{d}$. This is the approximation on which the analysis in Refs. $[10,11]$ was based. These papers comprise the most detailed studies of pion-nucleon scattering in the framework of heavy-baryon chiral perturbation theory (related studies have been published in [12-16]). It is obvious that one needs a precise description of the large isospin symmetric "background" of the scattering amplitude in order to be able to pin down the small isospin violating effects. The quality of the results obtained in Refs. $[10,11]$ makes us feel confident that we have a sufficiently accurate starting point.

In the second step, one should include the leading isospin breaking terms encoded in the pion and nucleon mass differences. The mass splitting for the nucleons amounts to about $1 \mathrm{MeV}$, whereas the charged- to neutral-pion mass difference is of the order of $5 \mathrm{MeV}$. To the accuracy we are working (the third order in small momenta and charges) one has to consider such effects. The strength of $\chi \mathrm{PT}$ now lies in the fact that one can consistently take into account only the effect from those isospin violating low-energy constants which enter the particles' masses. This is the approximation which 
we will consider here. In fact, in neutral-pion photoproduction off nucleons, to third order in small momenta, this approximation leads to the only isospin breaking effect, which reveals itself in the large cusp effect at the secondary threshold (i.e., at the $\pi^{+} n$ threshold in the case of $\gamma p \rightarrow \pi^{0} p$ ) [17].

In the third step, which goes beyond the scope of this work, one has to account for all virtual-photon effects, in particular soft-photon emission from charged-particle legs and the Coulomb poles due to the ladder exchange of (soft) virtual photons between charged external particles.

In the last two steps, the notion of partial waves with defined total isospin becomes doubtful. It is thus better not to give predictions for any specific isospin channel, but rather to consider quantities for measurable pion-nucleon reactions. After the third step has been performed, one will be able to directly fit to experimental cross section and polarization data. This will have the advantage that one will not have to rely on any code for electromagnetic corrections, which might or might not be compatible with the hadronic analysis. Instead, one will rather be able to compute electromagnetic corrections consistently in the framework of chiral perturbation theory. Fitting the low-energy constants (LEC's) of the full amplitude to experimental data will eventually allow us to pin down the values of the hadronic LEC's and to give an unambiguous definition of the hadronic phase shifts.

However, this work will only proceed up to the second step, the inclusion of mass difference effects and the leading strong isospin breaking vertices. We believe that the essential effects of isospin violation are captured in the calculation presented here. In [1], the size of isospin violation in threshold $\pi N$ scattering has been investigated. We now extend this analysis to the low-energy region above threshold; as in [1], we will quantify isospin breaking effects by using a set of relations, which are fulfilled in the limit of exact isospin symmetry.

This paper is organized as follows. In Sec. II we show how to generalize the pion-nucleon scattering amplitude in the case of isospin violation, and discuss how to quantify isospin breaking in terms of ratios that vanish in the limit of exact isospin symmetry. Section III is concerned with the calculation of the pertinent $\pi N$ scattering amplitude in the presence of strong and electromagnetic isospin breaking. We give results in Sec. IV and compare our approach to previous analyses of this topic. We conclude with a short summary and outlook in Sec. V.

\section{QUANTIFICATION OF ISOSPIN SYMMETRY BREAKING}

\section{A. Generalization of the scattering amplitude}

We consider the elastic scattering process

$$
\pi^{a}\left(q_{1}\right)+N\left(k_{1}\right) \rightarrow \pi^{b}\left(q_{2}\right)+N\left(k_{2}\right),
$$

where $\pi^{c}\left(q_{i}\right)$ denotes a pion state in the cartesian isospin basis with four-momentum $q_{i}$ and $N\left(k_{i}\right)$ a proton or a neutron with four-momentum $k_{i}$. The masses of the ingoing (outgoing) nucleon and pion are denoted by $m_{1(2)}$ and $M_{a(b)}$, respectively $\left(M_{1}=M_{2}=M_{\pi^{+}}, M_{3}=M_{\pi^{0}}\right)$. Consider the center-of-mass system (c.m.s.) with $\vec{k}_{i}=-\vec{q}_{i}(i=1,2)$. For the nucleons in the heavy-fermion approach, we set $k_{\mu}$ $=m v_{\mu}+p_{\mu}$ with $v \cdot p \ll m$, and $m$ the bare nucleon mass (i.e., the mass in the chiral limit with $e=0$ ). The small residual momentum $p_{\mu}$ is thus a quantity of chiral order 1 . The pion and the nucleon energy in the ingoing state are [we use $v=(1,0,0,0)]$

$$
\omega_{1}=v \cdot q_{1}=\sqrt{M_{a}^{2}+\vec{q}_{1}^{2}}, \quad E_{1}=\sqrt{m_{1}^{2}+\omega_{1}^{2}-M_{a}^{2}},
$$

in order. The energies of the outgoing pion and nucleon read

$$
\omega_{2}=\frac{\left(E_{1}+\omega_{1}\right)^{2}+M_{b}^{2}-m_{2}^{2}}{2\left(E_{1}+\omega_{1}\right)}, \quad E_{2}=E_{1}+\omega_{1}-\omega_{2},
$$

respectively. We come back to these kinematical relations after introducing the pion-nucleon scattering amplitudes appropriate for the discussion of isospin violation.

In the limit of isospin symmetry, pion-nucleon scattering can be fully described in terms of two amplitudes $T^{1 / 2}$ and $T^{3 / 2}$ or, equivalently, $T^{+}$and $T^{-}$. These amplitudes depend on two kinematical variables, which we can choose to be the pion c.m.s. energy $\omega$ and the invariant momentum transfer squared $t=\left(q_{1}-q_{2}\right)^{2}$. In that case, one cannot account for any difference in the scattering off protons compared to the one off neutrons. Indeed, there is no isospin operator which acts selectively on the nucleons. In the presence of isospin violation, i.e., isovector symmetry breaking terms such as $\left(m_{u}-m_{d}\right)(\bar{u} u-\bar{d} d)$, one thus has to generalize the standard form of the $\pi N$ scattering amplitude to

$$
\begin{aligned}
T^{b a}(\omega, t)= & \delta^{a b} T_{b a}^{+}(\omega, t)+\delta^{a b} \tau^{3} T_{b a}^{3+}(\omega, t)+i \epsilon^{b a c} \tau^{c} T_{b a}^{-}(\omega, t) \\
& +i \epsilon^{b a c} \tau^{c} \tau^{3} T_{b a}^{3-}(\omega, t)
\end{aligned}
$$

in terms of two isoscalar $\left(T_{b a}^{+, 3+}\right)$ and two isovector $\left(T_{b a}^{-, 3-}\right)$ amplitudes. It is important to realize that the $T_{b a}^{ \pm}$are exclusively sensitive to the neutral- to charged-pion mass difference. The $T_{b a}^{3 \pm}$ on the other hand distinguish between the different pion states, as well as between scattering off proton or neutron. Indeed the matrix $\tau^{3}$ acts differently on proton and neutron. Therefore, these amplitudes are also sensitive to the proton-neutron mass difference. The amplitudes are functions of two variables. As in the isospin symmetric case, we choose the mean pion energy $\omega$ and the invariant momentum transfer squared $t$. Since the total mass in the initial state is in general different from the mass of the outgoing particles, the energies of the pions in the ingoing and outgoing states, $\omega_{1,2}$, will also be no longer equal,

$$
\begin{aligned}
\Delta \omega & =\omega_{2}-\omega_{1} \\
& =\frac{\left(M_{b}^{2}-M_{a}^{2}\right)-\left(m_{2}^{2}-m_{1}^{2}\right)}{2 \sqrt{s}} \\
& =\frac{\left(M_{b}^{2}-M_{a}^{2}\right)-\left(m_{2}^{2}-m_{1}^{2}\right)}{2 m_{1}}\left[1-\frac{\omega_{1}}{m_{1}}+\mathcal{O}\left(q^{2}\right)\right],
\end{aligned}
$$


ith $\sqrt{s}$ the total c.m.s. energy. From Eq. (6) one can easily see that while the pion energies $\omega_{1,2}$ are of order $q$, their difference only starts out at second order in the chiral expansion. This has important consequences as will be discussed later. The $T$ amplitudes split into a spin-non-flip and a spinflip term, denoted by $g$ and $h$, respectively. The most general $\pi N$ scattering amplitude which will allow us to describe isospin symmetry violating effects thus reads

$$
\begin{aligned}
T_{\pi N}^{b a}= & \mathcal{N}_{1} \mathcal{N}_{2}\left\{\delta^{a b} g_{b a}^{+}+\delta^{a b} \tau^{3} g_{b a}^{3+}+i \epsilon^{b a c} \tau^{c} g_{b a}^{-}\right. \\
& +i \epsilon^{b a c} \tau^{c} \tau^{3} g_{b a}^{3-}+i \vec{\sigma} \cdot\left(\vec{q}_{2} \times \vec{q}_{1}\right) \\
& \left.\times\left[\delta^{a b} h_{b a}^{+}+\delta^{a b} \tau^{3} h_{b a}^{3+}+i \epsilon^{b a c} \tau^{c} h_{b a}^{-}+i \epsilon^{b a c} \tau^{c} \tau^{3} h_{b a}^{3-}\right]\right\}
\end{aligned}
$$

with $\mathcal{N}_{i}=\sqrt{\left(E_{i}+m_{i}\right) /\left(2 m_{i}\right)}(i=1,2)$ the standard spinor normalization [18]. We refrain from projecting these amplitudes onto states of defined total isospin, since isospin is no longer a good quantum number. But we can still define states with given angular and total momentum by

$$
\begin{aligned}
f_{l \pm, b a}^{( \pm / 3 \pm)}(s)= & \frac{\sqrt{E_{1}+m_{1}} \sqrt{E_{2}+m_{2}}}{16 \pi \sqrt{s}} \int_{-1}^{+1} d z \\
& \times\left[g_{b a}^{( \pm / 3 \pm)} P_{l}(z)+\vec{q}_{1} \cdot \vec{q}_{2} h_{b a}^{( \pm / 3 \pm)} \widetilde{P}_{l}(z)\right],
\end{aligned}
$$

with $\widetilde{P}_{l}(z)=P_{l \pm 1}(z)-z P_{l}(z)$ a combination of the usual Legendre polynomials. Isospin indices will now be combined in such a way as to match the physical reactions. Since the pions come in three and the nucleons in two charge states, we have ten reaction channels. In terms of the just defined amplitudes, these read

$$
\begin{gathered}
f_{\pi^{+} p \rightarrow \pi^{+} p}=f_{11}^{+}-f_{12}^{-}+f_{11}^{3+}-f_{12}^{3-}, \\
f_{\pi^{-} p \rightarrow \pi^{-} p}=f_{11}^{+}+f_{12}^{-}+f_{11}^{3+}+f_{12}^{3-}, \\
f_{\pi^{0} p \rightarrow \pi^{0} p}=f_{33}^{+}+f_{33}^{3+}, \\
f_{\pi^{+} n \rightarrow \pi^{+} n}=f_{11}^{+}+f_{12}^{-}-f_{11}^{3+}-f_{12}^{3-}, \\
f_{\pi^{-} n \rightarrow \pi^{-} n}=f_{11}^{+}-f_{12}^{-}-f_{11}^{3+}+f_{12}^{3-}, \\
f_{\pi^{0} n \rightarrow \pi^{0} n}=f_{33}^{+}-f_{33}^{3+}, \\
f_{\pi^{+} n \rightarrow \pi^{0} p}=-\frac{1}{\sqrt{2}}\left(f_{13}^{-}+f_{13}^{3-}\right)=f_{\pi^{0} p \rightarrow \pi^{+} n}, \\
f_{\pi^{-} p \rightarrow \pi^{0} n}=-\frac{1}{\sqrt{2}}\left(f_{13}^{-}-f_{13}^{3-}\right)=f_{\pi^{0} n \rightarrow \pi^{-} p},
\end{gathered}
$$

where, for simplicity of notation, we do not display the angular indices. Here we made use of the fact that $T_{11}^{+/ 3+}$ $=T_{22}^{+/ 3+}, T_{12}^{-13-}=T_{21}^{-13-}, T_{13}^{-}=T_{23}^{-}=T_{31}^{-}=T_{32}^{-}$, and $T_{13}^{3-}$ $=T_{23}^{3-}=-T_{31}^{3-}=-T_{32}^{3-}$. In Eqs. (15) and (16), we have also used time reversal invariance.

\section{B. Isospin relations}

Isospin violation is best characterized in terms of quantities which are exactly zero in the isospin limit of equal quark masses and vanishing electromagnetic coupling. We have eight reaction channels [see Eqs. (9)-(16)], which in the case of isospin symmetry are entirely described in terms of two amplitudes. One can thus write down six independent isospin relations (see also [19] for a general analysis)

$$
\begin{aligned}
& R_{1}=2 \frac{f_{\pi^{+} p \rightarrow \pi^{+} p}+f_{\pi^{-} p \rightarrow \pi^{-} p}-2 f_{\pi^{0} p \rightarrow \pi^{0} p}}{f_{\pi^{+} p \rightarrow \pi^{+} p}+f_{\pi^{-} p \rightarrow \pi^{-} p}+2 f_{\pi^{0} p \rightarrow \pi^{0} p}} \\
&=2 \frac{f_{11}^{+}-f_{33}^{+}+f_{11}^{3+}-f_{33}^{3+}}{f_{11}^{+}+f_{33}^{+}+f_{11}^{3+}+f_{33}^{3+}}, \\
& R_{2}=2 \frac{f_{\pi^{+} p \rightarrow \pi^{+} p}-f_{\pi^{-} p \rightarrow \pi^{-} p}-\sqrt{2} f_{\pi^{-} p \rightarrow \pi^{0} n}}{f_{\pi^{+} p \rightarrow \pi^{+} p}-f_{\pi^{-} p \rightarrow \pi^{-} p}+\sqrt{2} f_{\pi^{-} p \rightarrow \pi^{0} n}} 2 \frac{f_{12}^{-}-f_{13}^{-}+f_{12}^{3-}-f_{13}^{3-}}{f_{12}^{-}+f_{13}^{-}+f_{12}^{3-}+f_{13}^{3-}}, \\
& R_{3}=2 \frac{f_{\pi^{0} p \rightarrow \pi^{+} n}-f_{\pi^{-} p \rightarrow \pi^{0} n}}{f_{\pi^{0} p \rightarrow \pi^{+} n}+f_{\pi^{-} p \rightarrow \pi^{0} n}}=2 \frac{f_{13}^{3-}}{f_{13}^{-}}, \\
& R_{4}=2 \frac{f_{\pi^{+} p \rightarrow \pi^{+} p}-f_{\pi^{-} n \rightarrow \pi^{-} n}}{f_{\pi^{+} p \rightarrow \pi^{+} p}+f_{\pi^{-} n \rightarrow \pi^{-} n}}=2 \frac{f_{11}^{3+}-f_{12}^{3-}}{f_{11}^{+}-f_{12}^{-}}, \\
& R_{5}=2 \frac{f_{\pi^{-} p \rightarrow \pi^{-} p}-f_{\pi^{+} n \rightarrow \pi^{+} n}}{f_{\pi^{-} p \rightarrow \pi^{-} p}+f_{\pi^{+} n \rightarrow \pi^{+} n}}=2 \frac{f_{11}^{3+}+f_{12}^{3-}}{f_{11}^{+}+f_{12}^{-}}, \\
& R_{6}=2 \frac{f_{\pi^{0} p \rightarrow \pi^{0} p}-f_{\pi^{0} n \rightarrow \pi^{0} n}}{f_{\pi^{0} p \rightarrow \pi^{0} p}+f_{\pi^{0} n \rightarrow \pi^{0} n}}=2 \frac{f_{33}^{3+}}{f_{33}^{+}} .
\end{aligned}
$$

Note that this is just one of many possibilities to define these isospin ratios, but we find it particularly suitable. Again, we do not display angular variables. In the following, we will concentrate on the $S$ wave $\left(R_{i, 0+}\right)$ and two $P$ waves $\left(R_{i, 1 \pm}\right)$. The first two ratios, the so-called triangle relations, are based on the observation that in the isospin conserving case, the elastic scattering channels involving charged pions are trivially linked to the corresponding neutral-pion elastic scattering or the corresponding charge exchange amplitude. To be precise, all these ratios are to be formed with the real parts of the corresponding amplitudes. The imaginary parts of some of the amplitudes will be discussed later. Of particular interest is the second ratio, which is often referred to as the triangle relation. Only in this case have all three channels been measured (for pion kinetic energies as low as $30 \mathrm{MeV}$ in the center-of-mass system) and the 7\% strong isospin violation reported in $[6,7]$ refers to this ratio. We stress again that it is difficult to compare this number to the one we will obtain in our calculation since a very different method of separating the electromagnetic effects is used. The ratio $R_{6}$ parametrizes the large isospin violation effect for $\pi^{0}$ scattering off 
nucleons first found by Weinberg [2] and sharpened in [3], $R_{6} \simeq 25 \%$ at threshold. Note that in $R_{1}$, just as in $R_{6}$, the isovector terms drop out completely and one thus expects also a large isospin violation in this ratio (since the isoscalar parts are strongly suppressed and are of the same size as the symmetry breaking terms). To our knowledge, this particular ratio has been called attention to for the first time in [1]. From an experimental point of view, it has the advantage of avoiding the almost unmeasurable $n \pi^{0}$ amplitude appearing in $R_{6}$. On the other hand, as pointed out in Ref. [20], the elusive $\pi^{0} p$ scattering length might be measurable in neutral-pion photoproduction off protons below the $\pi^{+} n$ threshold. This results from the fact that the imaginary part of the electric dipole amplitude $E_{0+}$ is proportional to the respective $\pi N$ scattering length (final-state theorem); below the $\pi^{+} n$ threshold, one is thus exclusively sensitive to the small $\pi^{0} p$ phase shift. Since the two ratios $R_{1}$ and $R_{6}$ are entirely given in terms of isoscalar quantities, we expect large isospin symmetry breaking effects. But on the other hand, this also makes a precise determination of these ratios very difficult; indeed, the $S$-wave version of both these ratios is sensitive to the precise value of the combination of dimension-2 LEC's $2 c_{1}-c_{2}-c_{3}$, but the strong contribution to the isoscalar scattering length is not even known in sign at present. The predictions for the other ratios are more stable since they involve the larger (and better determined) isovector quantities. In what follows, we will calculate the six ratios $R_{i}$ to leading one-loop accuracy, i.e., to third order in small momenta. For that, we have to consider tree graphs, some with fixed coefficients and some with LEC's, and the leading one-loop graphs involving lowest-order couplings only.

\section{CALCULATION OF THE PION-NUCLEON AMPLITUDE}

As already mentioned, we will work to third order in the framework of heavy-baryon chiral perturbation theory. Since this method is well documented in the literature (see, e.g., the comprehensive review in [21]), we only discuss those terms in the isospin violating effective Lagrangian which are relevant for our analysis. For the particular case of isospin symmetric $\pi N$ scattering, a detailed exposition can be found in $[10,11]$.

In the purely mesonic sector, we have to take into account the following structures:

$$
\mathcal{L}_{\gamma^{*} \pi \pi}^{(2)}=\frac{F^{2}}{4}\left\langle u_{\mu} u^{\mu}+\chi_{+}\right\rangle+C\left\langle Q_{+}^{2}-Q_{-}^{2}\right\rangle,
$$

with

$$
Q=\frac{e}{2}\left(1+\tau_{3}\right)
$$

the nucleon charge matrix and $Q_{ \pm}$defined as follows:

$$
Q_{ \pm}=\frac{1}{2}\left(u^{\dagger} Q u \pm u Q u^{\dagger}\right)
$$

$F$ is the bare pion decay constant and the chiral vielbein is given by $u_{\mu}=i u^{\dagger} \partial_{\mu} U u^{\dagger}$. The scalar source $\chi$ includes the quark mass matrix, $\chi=2 B_{0} \mathcal{M}$, with $B_{0}$ being proportional to the vacuum expectation value of the scalar quark density. We work in the standard scenario of chiral symmetry breaking, $B_{0} \gg F$. From $\chi$ one constructs the fields $\chi_{ \pm}=u^{\dagger} \chi u^{\dagger}$ $\pm u \chi^{\dagger} u$. Furthermore, $\langle\cdots\rangle$ denotes the trace in isospin space. For the pion masses to leading order this gives

$$
\begin{gathered}
M_{0}^{2}=B_{0}\left(m_{u}+m_{d}\right), \\
M_{+}^{2}=B_{0}\left(m_{u}+m_{d}\right)+\frac{2}{F^{2}} e^{2} C .
\end{gathered}
$$

The experimentally known pion mass difference thus directly fixes the value of the low-energy constant $C, C=5.9$ $\times 10^{-5} \mathrm{GeV}^{4}$. Note that the pion mass difference up to second chiral order is of electromagnetic origin only.

In the one-nucleon sector, we take into account all terms related to strong isospin breaking as well as those electromagnetic terms which contribute to the nucleon masses. The relevant structures in the Lagrangian are [22]

$$
\begin{aligned}
\mathcal{L}_{\gamma^{*} \pi N}= & \bar{N}\left[i v \cdot D+c_{1}\left\langle\chi_{+}\right\rangle+c_{5} \tilde{\chi}_{+}+F^{2}\left(f_{1}\left\langle Q_{+}^{2}-Q_{-}^{2}\right\rangle\right.\right. \\
& \left.+f_{2}\left\langle Q_{+}\right\rangle Q_{+}+f_{3}\left\langle Q_{+}\right\rangle^{2}\right)+d_{17}\left\langle S \cdot u \chi_{+}\right\rangle \\
& \left.+i d_{18}\left[S \cdot D, \chi_{-}\right]+i d_{19}\left[S \cdot D,\left\langle\chi_{-}\right\rangle\right]+\cdots\right] N .
\end{aligned}
$$

Here, $D_{\mu}$ is the covariant derivative, $S_{\mu}$ the nucleon spin vector, and the $\tilde{\chi}$ denotes the traceless part of $\chi$ (for further definitions and the remaining isospin symmetric structures, see [10]). For the nucleon masses up to $\mathcal{O}\left(q^{2}\right)$ this means
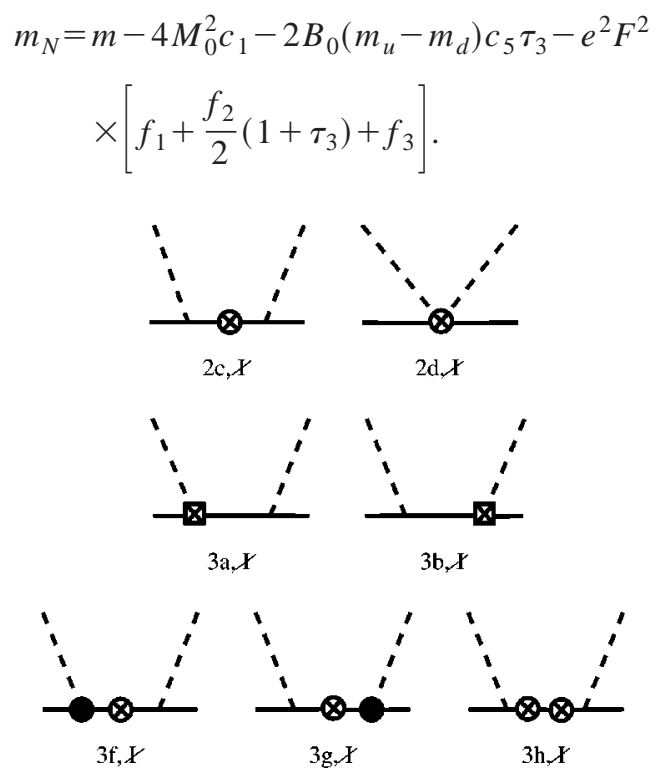

FIG. 1. Tree graphs contributing to isospin violation in $\pi N$ scattering. The circle-cross (box-cross) refers to a dimension-2 (-3) isospin violating vertex. The solid circle stands for an isospin symmetric vertex of second order. 

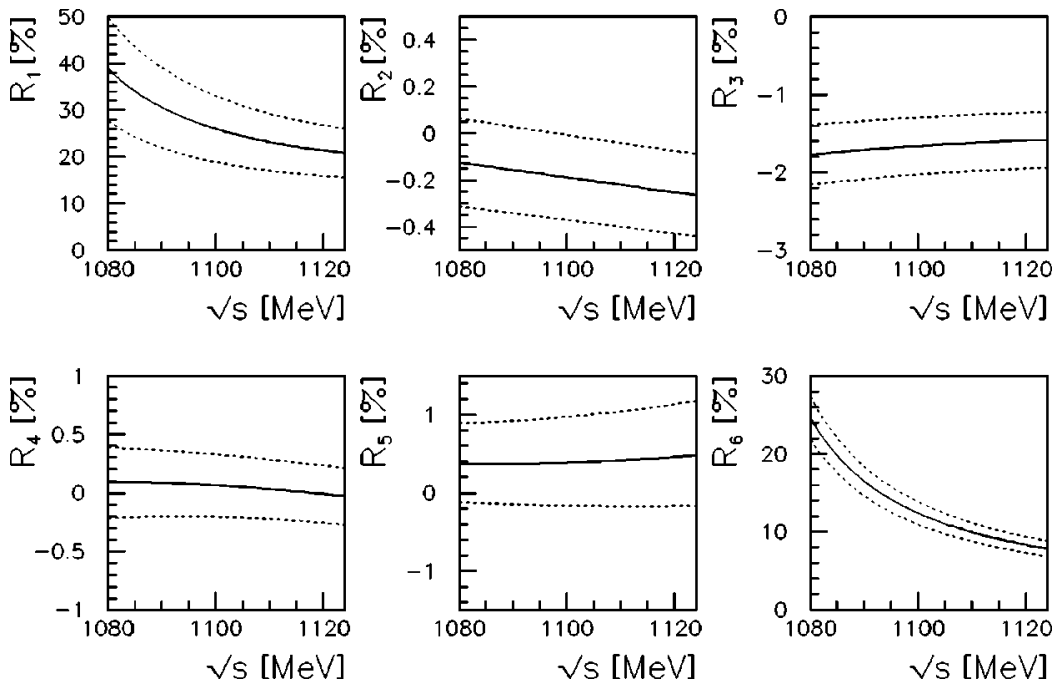

FIG. 2. Isospin violation in the $S$-wave projection of the ratios $R_{1}-R_{6}$. The dashed lines indicate the range for isospin violation if the parameters $f_{1}$ and $f_{2}$ are varied as discussed in the text.
The constant shift $-4 M_{0}^{2} c_{1}-e^{2} F^{2}\left(f_{1}+f_{2} / 2+f_{3}\right)$ is unobservable. The proton-to-neutron mass difference fixes the values of the LEC's through

$$
\begin{gathered}
\left(m_{p}-m_{n}\right)^{\mathrm{str}}=-4 B_{0}\left(m_{u}-m_{d}\right) c_{5}=(-2.05 \pm 0.30) \mathrm{MeV} \\
\left(m_{p}-m_{n}\right)^{\mathrm{em}}=-e^{2} F^{2} f_{2}=(0.76 \mp 0.30) \mathrm{MeV}
\end{gathered}
$$

These values are taken from [23], where the electromagnetic mass shift was calculated with the help of the Cottingham formula. Equation (30) directly fixes the value of the electromagnetic coupling $f_{2}, f_{2}=(-0.45 \pm 0.19) \mathrm{GeV}^{-1}$. We then fix $B_{0}\left(m_{u}-m_{d}\right) c_{5}$ in such a way that the neutron-proton mass difference coincides with the experimentally determined value. We want to stress that, contrary to the pion case, the nucleon mass difference is of electromagnetic and strong nature. Besides $c_{1}$, also $c_{5}, f_{1}$, and $f_{2}$ contribute to the $\pi \pi N N$ vertex which is given by

$$
\begin{aligned}
& -i \frac{4}{F^{2}} c_{1} M_{0}^{2} \delta^{a b}-i c_{5} B_{0}\left(m_{u}-m_{d}\right) \frac{1}{F^{2}} \\
& \quad \times\left[2 \delta^{a b} \delta^{a 3}+i \epsilon^{b a c} \tau^{c}\left(\delta^{a 3}-\delta^{b 3}\right)\right] \tau^{3} \\
& -i e^{2}\left[2 f_{1} \delta^{a b}\left(1-\delta^{a 3}\right)+\frac{f_{2}}{4}\left[2 \delta^{a b} \tau^{3}\left(1-\delta^{a 3}\right)\right.\right. \\
& \left.\left.\quad+i \epsilon^{b a c} \tau^{c} \tau^{3}\left(\delta^{b 3}-\delta^{a 3}\right)\right]\right]
\end{aligned}
$$

Whereas $c_{5}$ and $f_{2}$ can be fixed through Eqs. (29) and (30), respectively, the value of $f_{1}$ is unknown. In our analysis, it will be varied in some reasonable range. Let us estimate these bounds by observing that the $f_{1}$ contribution to the nucleon mass shift should be of the same order of magnitude as any photonic loop, and thus

$$
e^{2} F_{\pi}^{2} f_{1} \sim\left(\frac{e}{4 \pi}\right)^{2} m_{N}
$$

where $F_{\pi}$ is the physical pion decay constant. This leads to $f_{1} \sim 1 \mathrm{GeV}^{-1}$ as a natural order. ${ }^{1}$ In the following, we will use the range $e^{2} f_{1}=0.0 \pm 0.1 \mathrm{GeV}^{-1}$. We will also need the values of the counterterms related to isospin symmetric structures. The LEC's $c_{i}$ and $d_{i}$ are taken from fits 1, 2, 3 of [10]. These are fits of the third-order isospin symmetric amplitude to the low-energy pion-nucleon phase shift analyses of Refs. [24,25,26], respectively.

In terms of the operators defined in Eqs. (23) and (27), retaining only the terms leading to the strong and electromagnetic hadron masses is achieved by setting

$$
C e^{2} \neq 0, \quad f_{i} e^{2} \neq 0, \quad m_{u}-m_{d} \neq 0, \quad \text { but } \quad e^{2}=0 .
$$

The isospin symmetric tree graphs calculated to first, second, and third order, as well as the loop diagrams can be found in [15]. The additional tree graphs with explicit isospin violating vertices $\left(\sim f_{1}, f_{2}, \sim c_{5}, \sim \bar{d}_{17}, \bar{d}_{18}, \bar{d}_{19}\right)$ are shown in Fig. 1. Before giving results, some important remarks concerning the chiral power counting are in order. Although the socalled Weinberg-Tomozawa $\bar{N} N \pi \pi$ contact graph gives a first-order contribution to $g_{b a}^{-}$, in the ratios $R_{i}$ its effect is always proportional to $\Delta \omega$, which is of second order; see Eq. (6). Consequently, isospin violation only starts at second order in the chiral expansion. The chiral power counting is not as straightforward in the case of isospin violation as in the isospin symmetric case. The difference of the pion energies is of second order, and should thus be neglected whenever it only leads to contributions of $\mathcal{O}\left(q^{4}\right)$. However, if this was done systematically, a diagram, which in fact vanishes at threshold, would now give a finite contribution. This can easily be seen in the following example: At threshold, either the incoming or the outgoing pion are at rest, and thus $\vec{q}_{1}$ - $\vec{q}_{2}=0$. In a strict chiral expansion, this should be replaced by $\left(2 \omega^{2}-M_{a}^{2}-M_{b}^{2}-t\right) / 2$, which does not vanish for $M_{a}$ $\neq M_{b}$. In order to avoid such spurious effects, we decided

\footnotetext{
${ }^{1}$ This dimensional analysis differs from the one of [9], which considers $f_{1} \sim 1 /(4 \pi) \mathrm{GeV}^{-1}$ as a natural order of magnitude.
} 

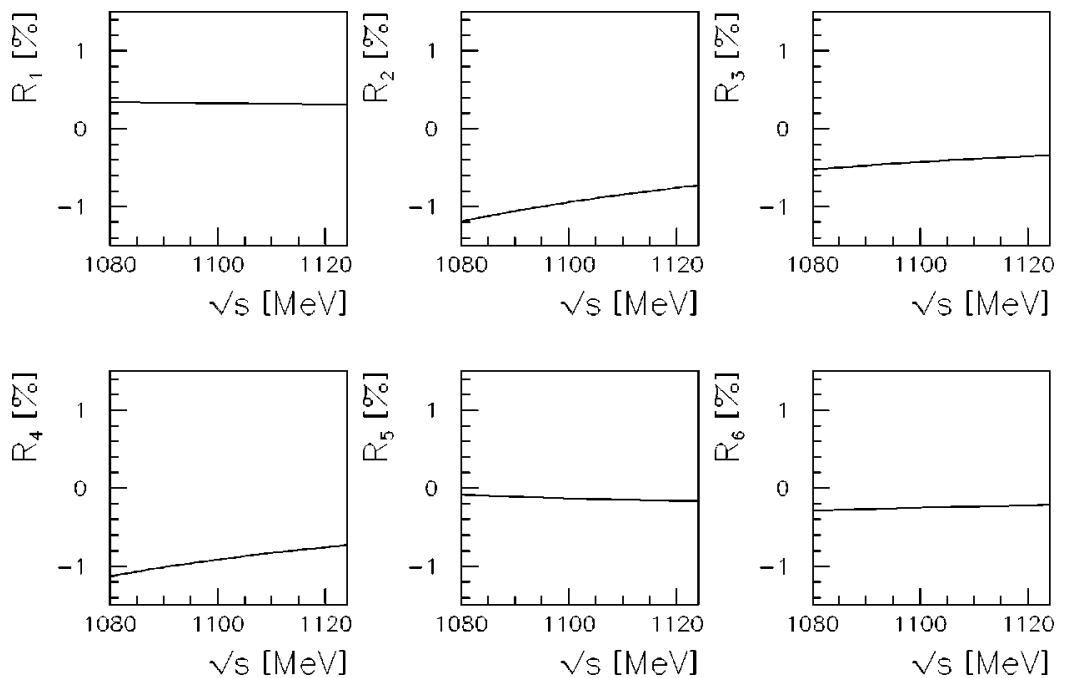

FIG. 3. Isospin violation in the $P_{3}$-wave projection of the ratios $R_{1}-R_{6}$. also to account for $\Delta \omega$ in some cases where it leads to a higher-order effect, giving priority to the correct description of the amplitude at threshold. This is of course fully legitimate, since we only intend to give the amplitude correctly up to third order in the small expansion parameter $q$.

\section{RESULTS}

In this section, we want to generalize the analysis of Ref. [1] to the energy range above threshold. We also give some additional threshold results not shown in that paper.

\section{A. Momentum dependence}

Above threshold, we need the spin-non-flip amplitudes $g^{ \pm, 3 \pm}$ and the spin-flip ones $h^{ \pm, 3 \pm}$. The expressions for the amplitudes are given in [27]. We will focus on the results from fit 1 (based on the KA85 phase shifts). This analysis is different from the one in Ref. [1] in one important point: In [1], different reactions were compared at their particular threshold energy; this means that the ratios were formed by combining amplitudes at different center-of-mass energies. This will not be the case anymore. We will always consider $\sqrt{s}$ as a fixed quantity when forming ratios. This explains the difference between the numbers at low energy obtained here and the ones presented in [1].

In Fig. 2, we show the isospin breaking effects on the $S$ waves for the different ratios $R_{1}-R_{6}$, as a function of the total center-of-mass energy $\sqrt{s}$. Note that in these calculations, the spinor normalization factors are also taken into account. ${ }^{2}$ As expected, the purely isoscalar ratios $R_{1}$ and $R_{6}$ show very large isospin breaking effects. Those ratios, on the other hand, which are given in terms of both isoscalar and isovector quantities, show a rather small effect, on the order

\footnotetext{
${ }^{2}$ This is in contrast to what was done in [1], but the inclusion of these factors is necessary if one wants to define the partial waves in the standard way. We have checked that the conclusions concerning isospin violation do not depend on whether we include this factor or not.
}

of $0-2 \%$. Also, the energy dependence is as expected: the lower the energy, the more important the quark mass difference is compared to the kinetic energies of the particles, and the larger the isospin breaking effects should be. As well for $R_{1}$ as for $R_{6}$, the effect drops by a factor of 2 over the first $50 \mathrm{MeV}$. The ratios $R_{2}-R_{5}$ remain almost constant over the whole energy range displayed here. The previously discussed theoretical uncertainties due to the lack of knowledge of $f_{1}$ and $f_{2}$ lead to the bands displayed in Fig. 2. ${ }^{3}$ It is important to note that the variation in $f_{1}$ only shows up in $R_{1}, R_{4}$, and $R_{5}$. Whereas the range in $f_{1}$ dominates the uncertainty in $R_{1}$, it leads to vanishingly small contributions to $R_{4}$ and $R_{5}$. It is thus the uncertainty in $f_{2}$ which largely dominates the width of the bands in $R_{2}-R_{6}$. Since electromagnetic effects proportional to $f_{1}$ and $f_{2}$ do not contribute to neutral-pion scattering, the band for $R_{6}$ is entirely due to the variation in $B_{0}\left(m_{u}-m_{d}\right) c_{5}$. We see that the prediction for $R_{1}$ is quite sensitive to the precise value of the parameter $f_{1}$. But the conclusions remain unchanged: we expect huge isospin violation in the $S$-wave parts of the purely isoscalar ratios $R_{1}$ and $R_{6}$.

The corresponding $P_{3^{-}}$and $P_{1}$-wave projections of these relations are shown in Figs. 3 and 4. The notation is standard: $l=1$ for both $P$ waves, for $P_{3}$ the total angular momentum is $3 / 2$ whereas $P_{1}$ is related to $j=1 / 2$. For $P_{3}$, everything is as expected: isospin breaking is small, as well in the purely isoscalar channels as in the ones given by isoscalar and isovector quantities. It is interesting to note that in this projection, $R_{2}$ turns out to show the biggest effect, about $-1.2 \%$ close to threshold. However, for the $P_{1}$ waves, the results are more surprising: indeed for $R_{2}$ and for $R_{3}$, the effects from isospin violation become larger with increasing center-of-mass energy: $50 \mathrm{MeV}$ above threshold, they are as big as $15-20 \%$. But we run into the same problems as in the threshold analysis [1]: namely, that the denominators of

\footnotetext{
${ }^{3} \mathrm{We}$ stress again that a variation of $f_{2}$ entails a variation of $B_{0}\left(m_{u}-m_{d}\right) c_{5}$ such that the neutron-proton mass difference remains constant.
} 

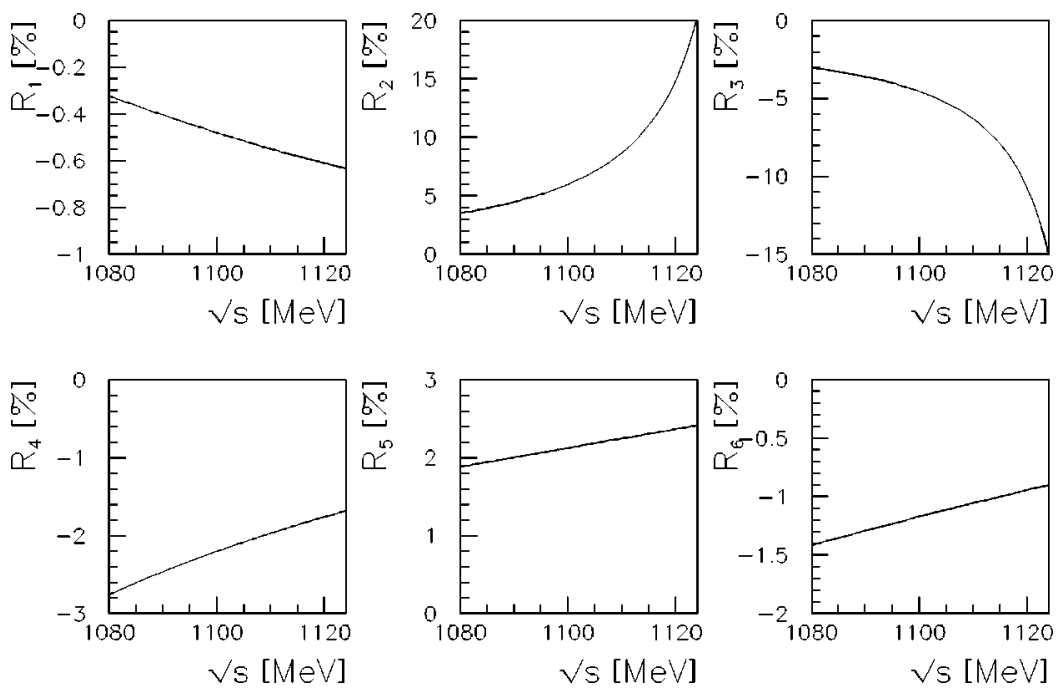

FIG. 4. Isospin violation in the $P_{1}$-wave projection of the ratios $R_{1}-R_{6}$. some of the relations become very small in the $P_{1}$ projection. We find a really spectacular result indicating isospin violation as big as $30 \%$ in $R_{2}$ and $R_{3}$, but it will be a hopeless case to measure such effects, since the associated isospin conserving part is vanishingly small as well. It is thus necessary to find a different representation, to project out different quantities than the usual partial waves. From Eq. (8) we obtain

$$
\begin{gathered}
P_{3}(s)=\left|\vec{q}_{1}\right|\left|\vec{q}_{2}\right|[\mathcal{G}(s)+\mathcal{H}(s)], \\
P_{1}(s)=\left|\vec{q}_{1}\right|\left|\vec{q}_{2}\right|[\mathcal{G}(s)-2 \mathcal{H}(s)],
\end{gathered}
$$

with

$$
\begin{gathered}
\mathcal{G}(s)=\frac{\sqrt{E_{1}+m_{1}} \sqrt{E_{2}+m_{2}}}{16 \pi \sqrt{s}} \frac{1}{\left|\vec{q}_{1}\right|\left|\vec{q}_{2}\right|} \int_{-1}^{+1} d z g_{b a} P_{1}(z), \\
\mathcal{H}(s)=\frac{\sqrt{E_{1}+m_{1}} \sqrt{E_{2}+m_{2}}}{16 \pi \sqrt{s}} \int_{-1}^{+1} d z h_{b a}\left(P_{2}(z)-z P_{1}(z)\right) .
\end{gathered}
$$

In Figs. 5 and 6, we thus show isospin breaking in the ratios $R_{1}-R_{6}$ formed with the quantities $\mathcal{G}$ and $\mathcal{H}$, respectively. From these figures we can see that indeed there is no large isospin symmetry breaking in the $P$ waves. The problems for the representation in terms of $P_{1}$ and $P_{3}$ only resulted from very small denominators. Interestingly, the ratios $R_{1}$ and $R_{6}$, which were found to give large results in the $S$-wave projection, are now found to be very small. Their $\mathcal{G}$ and $\mathcal{H}$ projections show an effect much smaller than $1 \%$. Note that neither the variation of $f_{1}$ nor the combined one of $f_{2}$ and $c_{5}$ affects the $P$-wave projections of our ratios.

In Fig. 7, we show an analog representation of isospin breaking effects to what was presented in [7]. The plot is based on the philosophy put forward in [28]. Provided that isospin is a good symmetry, the entire information concerning the elastic $\pi^{ \pm} p$ and the charge exchange $\pi^{-} p \rightarrow \pi^{0} n$ reactions is contained in two energy-dependent complex functions per partial wave, the isosymmetric $f^{+}$and isoasymmetric $f^{-}$. In such a case, the $\pi^{+} p$ reaction is described by $f^{+}-f^{-}$(represented by the vertical band in Fig. 7 ), the $\pi^{-} p$ reaction by $f^{+}+f^{-}$(horizontal band in Fig. 7), and the charge exchange reaction is given by $-\sqrt{2} f^{-}$
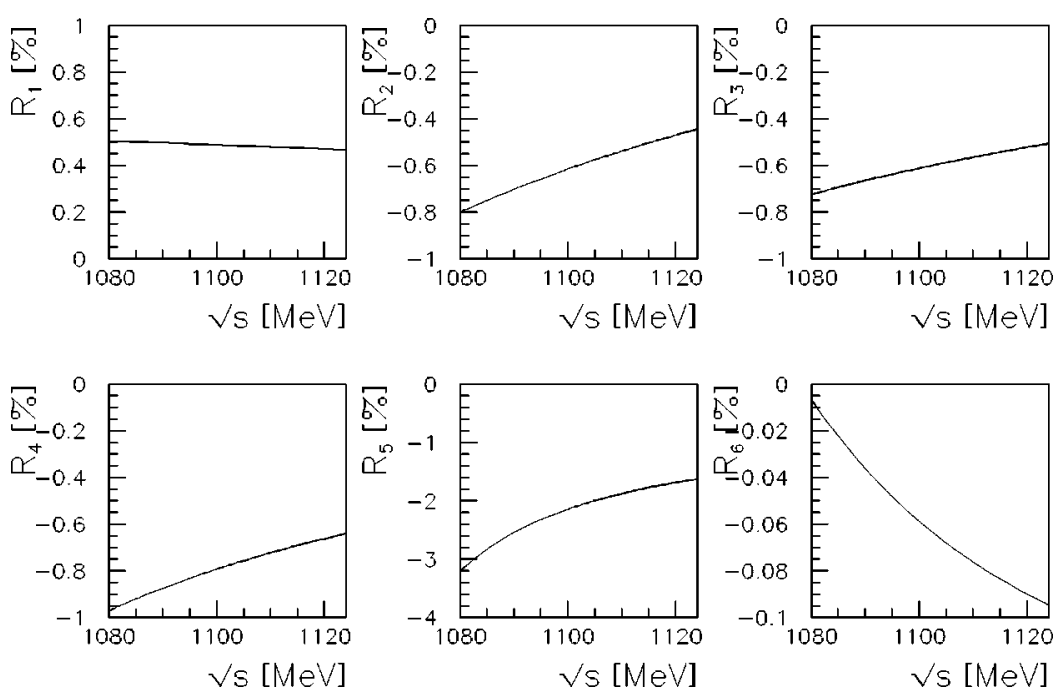

FIG. 5. Isospin violation in the $\mathcal{G}$ projection of the ratios $R_{1}-R_{6}$. 

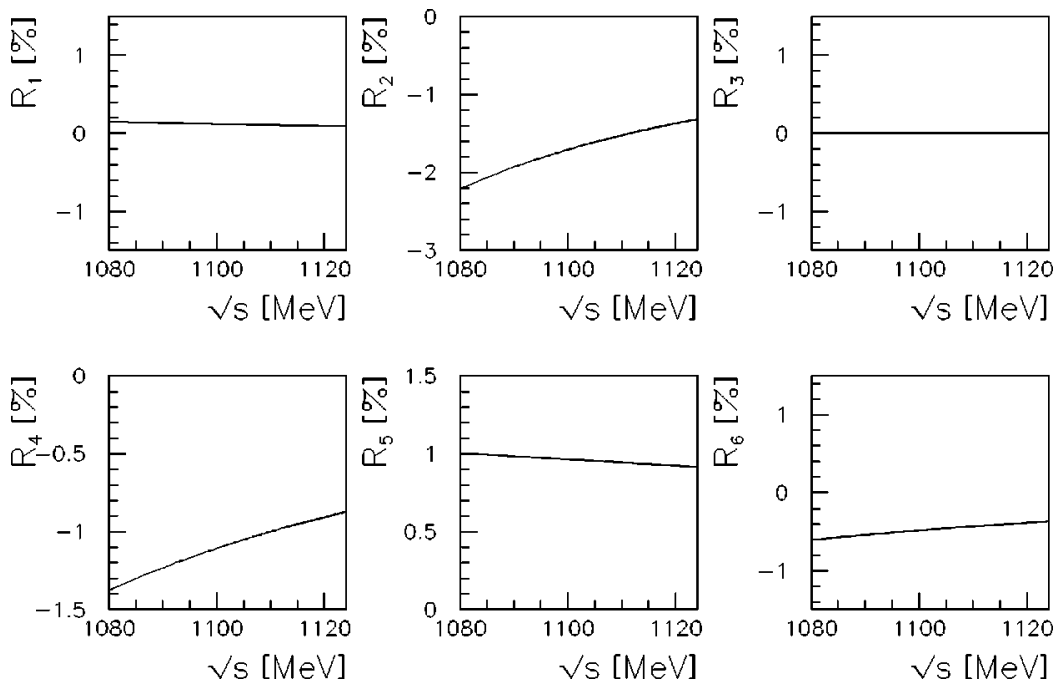

FIG. 6. Isospin violation in the $\mathcal{H}$ projection of the ratios $R_{1}-R_{6}$. (shown by the diagonal band in Fig. 7). At any energy, the principle of isospin symmetry necessitates a common overlap of the three bands. Here, we only show the corresponding bands at threshold. Note that most of the bands are degenerate to a line, since we have not taken into account any experimental errors, but only the spread due to the lack of knowledge of the value of $f_{1}$. In this way of looking at the problem, we cannot quantify isospin violation precisely, since we do not have any experimental errors, which would allow us, as in the case of [7], to give the standard deviation of the difference between the charge exchange band and the intersection of the two elastic bands.

\section{B. Threshold analysis}

The threshold region was already discussed in Ref. [1]; in particular the large effect on the ratio $R_{1}$ was for the first time pointed out in that paper and the influence of the operator $\sim c_{5}$ was analyzed. In that paper, predictions for pion scattering off protons were given. For completeness, in Table I, we collect these predictions together with the ones for scattering off neutrons (for the central values of the LEC's $c_{5}, f_{1}$, and $f_{2}$ ). The large difference among the three sets of predicted values comes from the various input data; in particular, the theoretically most interesting scattering lengths for neutral-pion scattering off nucleons cannot be predicted very reliably. This theoretical uncertainty calls for a dedicated pion photoproduction experiment $\gamma p \rightarrow \pi^{0} p$ below the secondary threshold which will allow one to pin down the scattering length $a\left(\pi^{0} p\right)$, as detailed in Ref. [20]. For a more precise discussion on isospin violation effects in threshold $S$ waves, see [1].

\section{Comparison to other analyses}

The question of isospin violation in pion-nucleon scattering has stimulated vast efforts on the experimental, but also on the phenomenological side.

Gibbs, Ai, and Kaufmann [6] restrict their analysis to the triangle relation (arguing that only in this channel, all reactions are experimentally accessible) and look for deviations from zero of $D=f_{\mathrm{CEX}}-\left(f_{\pi^{+}{ }_{p}}-f_{\pi^{-} p}\right) / \sqrt{2}$. They are only interested in isospin violation which lies beyond effects due to the hadronic mass differences and the Coulomb corrections. They thus describe different physics than we do, and our results should consequently not be directly compared to theirs. They find $D=(-0.012 \pm 0.003) \mathrm{fm}$, thus a $4 \sigma$ effect. They cannot conclude where the breaking actually occurs; it could as well be in the charge exchange, in the elastic amplitudes, or in a combination of both.

Matsinos [7] analyzes the whole set of low-energy pionnucleon scattering data with the help of an extended tree level model [29]. The known electromagnetic corrections and hadronic mass effects are taken care of by the NORDITA method [30]. He finds consistency of the low-energy pion-nucleon database and comes to similar conclusions than Gibbs et al.: the relative difference in the real parts of the two $S$-wave amplitudes (the one of the elastic channels and
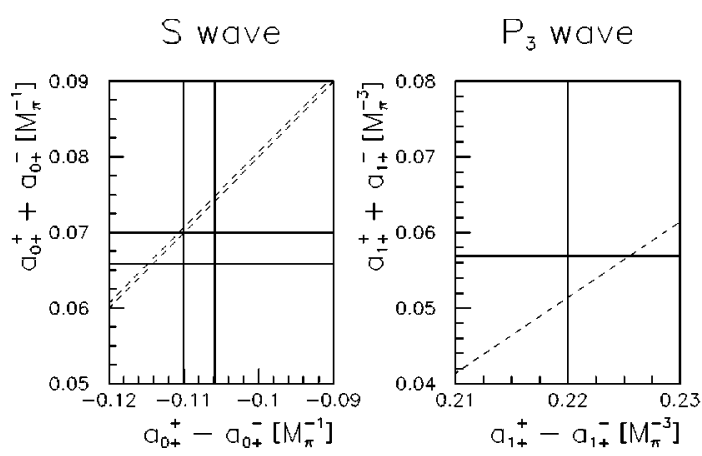

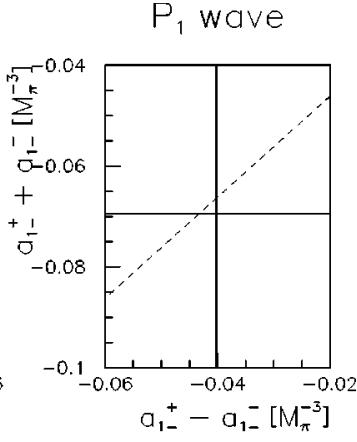

FIG. 7. Real parts of the scattering lengths and volumes. The $\pi^{+} p$ elastic process is represented by the vertical bands, the $\pi^{-} p$ elastic process by the horizontal bands, and the charge exchange reaction $\pi^{-} p \rightarrow \pi^{0} n$ by the diagonal bands. 
TABLE I. Values of the scattering lengths for pion scattering off nucleons in units of $10^{-3} / M_{\pi^{+}}$for the various parameter sets as given by fits 1, 2, 3 of Ref. [10].

\begin{tabular}{lccc}
\hline \hline & Fit 1 & Fit 2 & Fit 3 \\
\hline$a\left(\pi^{+} p \rightarrow \pi^{+} p\right)$ & -108.8 & -83.8 & -95.3 \\
$a\left(\pi^{-} p \rightarrow \pi^{-} p\right)$ & $70.5+i 3.7$ & $71.3+i 3.7$ & $76.9+i 3.7$ \\
$a\left(\pi^{0} p \rightarrow \pi^{0} p\right)$ & -13.4 & -0.1 & -2.6 \\
$a\left(\pi^{+} n \rightarrow \pi^{+} n\right)$ & $69.7+i 3.7$ & $70.5+i 3.7$ & $76.2+i 3.7$ \\
$a\left(\pi^{-} n \rightarrow \pi^{-} n\right)$ & -109.6 & -84.6 & -96.1 \\
$a\left(\pi^{0} n \rightarrow \pi^{0} n\right)$ & -11.0 & 2.2 & -0.2 \\
$a\left(\pi^{-} p \rightarrow \pi^{0} n\right)$ & -125.7 & -108.5 & -120.8 \\
$a\left(\pi^{0} p \rightarrow \pi^{+} n\right)$ & $-124.9-i 0.6$ & $-107.8-i 0.6$ & $-119.9-i 0.6$ \\
\hline \hline
\end{tabular}

the one corresponding to charge exchange) amounts to (6.4 $\pm 1.4) \%$.

Both authors are only interested in isospin violation stemming from strong vertices, neither in electromagnetic corrections to the amplitudes nor in mass difference effects. This approach is completely different from ours and the results should not be naively compared. Both analyses come to the same conclusions, although they are based on different models for describing the strong part of the $\pi N$ interaction and they use different algorithms for separating strong effects from electromagnetic and mass difference ones. But we stress again that in the framework we are using, a consistent separation of the electromagnetic and the strong effects is possible and to our knowledge this has not been achieved before. Only when a mapping of the method developed here on the commonly used procedures of separating electromagnetic and hadronic mass effects (such as the NORDITA method [30]) has been performed will a sensible comparison with the numbers quoted in the literature be possible. In order to achieve this, further work in chiral perturbation theory calculations will have to be done.

In meson exchange models, isospin violation in pionnucleon scattering is mainly accounted for by $\pi \eta$ mixing. Pion-nucleon scattering then proceeds, e.g., through an $\eta$ production process followed by an isospin symmetry breaking transition $\eta \rightarrow \pi^{0}$. This process can only occur if at least one of the pions is neutral. In an $\mathrm{SU}(2)$ approach to chiral perturbation theory, $\pi \eta$ mixing is hidden in some counterterms. If one performs an $\mathrm{SU}(3)$ calculation, one can explicitly take into account such effects, and one can convince oneself that they are indeed proportional to $m_{u}-m_{d}[23,31]$. Another process which could explain isospin violation is $\rho \omega$ mixing [32]. Although this effect can account for the same magnitude of isospin breaking as observed in [6] and [7], it gives the wrong sign. Both diagrams can be seen in Fig. 8 .

\section{SUMMARY}

We have presented an analysis of isospin breaking effects in pion-nucleon scattering due to the light quark mass differ-
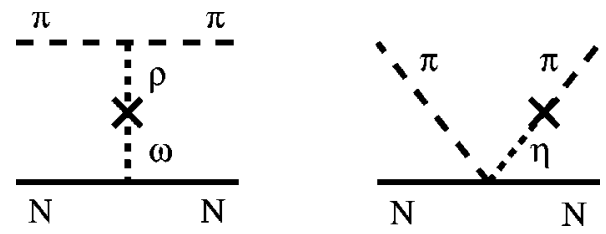

FIG. 8. Feynman diagrams commonly used in meson exchange models to explain isospin violation in pion-nucleon scattering.

ence and the dominant virtual-photon effects.

(i) We have taken into account all operators related to strong isospin breaking and the electromagnetic ones that lead to the pion and nucleon mass differences. Stated differently, the finite parts of some of the virtual-photon operators contributing at this order have been set to zero. This allows one in particular to isolate the contribution of the strong isospin breaking dimension-2 isovector operator first considered by Weinberg. We have considered a set of six ratios $R_{i}$, which vanish in the limit of isospin conservation. From these, four involve isovector and isoscalar amplitudes $\left(R_{2,3,4,5}\right)$ while the two others are of purely isoscalar type $\left(R_{1,6}\right)$.

(ii) We have extended the analysis of Ref. [1] to higher center-of-mass energies. In the $S$ wave, isospin violating effects tend to disappear rather quickly in energy. But in the $P_{1}$ wave, as a result of a very small isospin symmetric part, relative isospin violation becomes very large in some ratios. In order to give a more reliable description of the phenomenon, we presented isospin breaking in two other quantities, which are more directly related to the spin-flip and spin-nonflip amplitudes. We conclude that isospin violation effects are small in these new projections.

(iii) We have tabulated the theoretical predictions for $S$-wave scattering lengths in the eight physical channels and stressed the importance of measuring the elusive $\pi^{0} p$ channel via precise photoproduction experiments (which should be feasible at MAMI or the TUNL-FELL).

We want to note again that within the framework presented here, a unique and unambiguous separation of all different isospin violating effects is possible. To access the size of isospin violation encoded in the presently available pionnucleon scattering data, an extension of this scheme to include hard and soft photons is mandatory. Once this is done, it will be possible to analyze the cross section data directly without recourse to any model for separating electromagnetic or hadronic mass effects, thus avoiding any mismatch by combining different approaches or models.

\section{ACKNOWLEDGMENTS}

We would like to thank Aron Bernstein for very stimulating discussions. 
[1] N. Fettes, Ulf-G. Meißner, and S. Steininger, Phys. Lett. B 451, 233 (1999).

[2] S. Weinberg, Trans. NY Acad. Sci. 38, 185 (1977).

[3] Ulf-G. Meißner and S. Steininger, Phys. Lett. B 419, 403 (1998).

[4] S. Weinberg, in Chiral Dynamics: Theory and Experiment, edited by A. M. Bernstein and B. R. Holstein (SpringerVerlag, Berlin, 1995).

[5] U. van Kolck, Ph.D. thesis, University of Texas at Austin, 1993 (unpublished).

[6] W. R. Gibbs, Li Ai, and W. B. Kaufmann, Phys. Rev. Lett. 74, 3740 (1995).

[7] E. Matsinos, Phys. Rev. C 56, 3014 (1997).

[8] Ulf-G. Meißner, G. Müller, and S. Steininger, Phys. Lett. B 406, 154 (1997); 407, 454(E) (1997).

[9] G. Müller and Ulf-G. Meißner, Nucl. Phys. B556, 265 (1999).

[10] N. Fettes, Ulf-G. Meißner, and S. Steininger, Nucl. Phys. A640, 199 (1998).

[11] N. Fettes and Ulf-G. Meißner, Nucl. Phys. A676, 311 (2000).

[12] V. Bernard, N. Kaiser, and Ulf-G. Meißner, Phys. Lett. B 309, 421 (1993).

[13] V. Bernard, N. Kaiser, and Ulf-G. Meißner, Phys. Rev. C 52, 2185 (1995).

[14] V. Bernard, N. Kaiser, and Ulf-G. Meißner, Nucl. Phys. A619, 261 (1997).

[15] M. Mojžiš, Eur. Phys. J. C 2, 181 (1998).

[16] P. Büttiker and Ulf-G. Meißner, Nucl. Phys. A668, 97 (2000).

[17] V. Bernard, N. Kaiser, and Ulf-G. Meißner, Z. Phys. C 70, 483 (1996).
[18] S. Steininger, Ulf-G. Meißner, and N. Fettes, J. High Energy Phys. 09, 008 (1998).

[19] W. B. Kaufmann and W. R. Gibbs, Ann. Phys. (N.Y.) 214, 84 (1992).

[20] A. M. Bernstein, Phys. Lett. B 442, 20 (1998).

[21] V. Bernard, N. Kaiser, and Ulf-G. Meißner, Int. J. Mod. Phys. E 4, 193 (1995).

[22] S. Steininger, Ph.D. thesis, University of Bonn, 1999 (unpublished).

[23] J. Gasser and H. Leutwyler, Phys. Rep. 87, 77 (1982).

[24] R. Koch, Nucl. Phys. A448, 707 (1986); Z. Phys. C 15, 161 (1982).

[25] E. Matsinos, hep-ph/9807395; private communication.

[26] R. A. Arndt et al., SAID on-line program; see website http:// said.phys.vt.edu.

[27] N. Fettes, Ph.D. thesis, University of Bonn, 2000 (unpublished).

[28] A. Badertscher et al., Zürich Report No. ETHZ-IPP 94-10 (1994); H.-J. Leisi et al., in Chiral Dynamics: Theory and Experiment [4].

[29] P. F. A. Goudsmit, H.-J. Leisi, E. Matsinos, B. L. Birbrair, and A. B. Gridnev, Nucl. Phys. A575, 673 (1994).

[30] J. Hamilton, B. Tromberg, and I. Фverbrø, Nucl. Phys. B60, 443 (1973); B. Tromberg and J. Hamilton, ibid. B76, 483 (1974); B. Tromberg, S. Waldenstrøm, and I. Øverbrø, Phys. Rev. D 15, 725 (1977).

[31] B. L. Ioffe and M. A. Shifman, Phys. Lett. 95B, 99 (1980).

[32] B. L. Birbrair and A. B. Gridnev, Phys. Lett. B 335, 6 (1994). 